\title{
Hip Arthroplasty in Two Patients With Congenital Pubic Diastasis and Severe Dysplastic Hips
}

Christian Götze ( $\nabla$ christian.goetze@muehlenkreiskliniken.de )

Auguste-Viktoria Klinikum https://orcid.org/0000-0000-0000-0001

Christian-Dominik Peterlein

Auguste-Viktoria Hospital: Auguste-Viktoria-Klinik

Research article

Keywords: Congenital pubic diastasis, hip dysplasia, cementless total hip arthroplasty

Posted Date: January 20th, 2021

DOI: https://doi.org/10.21203/rs.3.rs-150238/v1

License: (9) This work is licensed under a Creative Commons Attribution 4.0 International License. Read Full License 


\title{
Hip arthroplasty in two patients with congenital pubic diastasis and severe dysplastic hips-
}

\author{
Goetze, Christian; Peterlein, Christian-Dominik
}

Götze, Christian

Clinic for orthopedics

Auguste-Viktoria Clinic,

Ruhr University Bochum,

Am Kokturkanal 2,

32545 Bad Oeynhausen, Germany

christian.goetze@muehlenkreiskliniken.de

Peterlein, Christian-Dominik

Clinic for orthopedics

Auguste-Viktoria Clinic,

Ruhr University Bochum,

Am Kokturkanal 2,

32545 Bad Oeynhausen, Germany

christian-dominik.peterlein@muehlenkreiskliniken.de 
Abstract

Background: Congenital pubic diastasis in combination with a dysplastic situation of the hip is a rather rare malformation. Two cases of young female patients prompted us to report the results of total hip arthroplasty (THA) due to secondary osteoarthritis. Our treatment strategy and short- as well in one case long-term results are described in regard to the rather rare literature.

Methods: We describe a case of a 39 -year-old female patient with a pubic diastase and severe hip dysplasia on both sides treated with a primary total hip arthroplasty (THA). High overweight of more than 275 pounds, diabetes mellitus and multiple prior operation aggravate the operative procedure. Main problem based on the fixation strategy of the cup in this retroverted acetabulum presented in CT-analysis with elevated rotation center due to the dysplastic situation. In the other case a 52-year old female lady presented dysplastic osteoarthritis of the left hip.

Results: In the first case both hips were treated with a cementless osteoconductive cup (TM, Zimmer-Biomet) and a cementless stem (Alloclassic SL, Zimmer-Biomet). A $10^{\circ}$ elevated rim liner of the cup was used in order to avoid dislocation. Both hips were 9 and 8.5 years postoperative radiologically still in place without any signs of loosening. There was an improvement of quality of life documentated with an amelioration of the Harris Hip Score from preoperative 14 points to 68 points. In the second case a conventional hemispherical cup (Alloclassic-Allofit, Zimmer-Biomet) was placed in the retroverted acetabulum combined with a cementless stem (Fitmore A, Zimmer-Biomet) attached at the metaphseal proximal femur bone. 6 months postoperative, the patient was free of symptoms with an increased quality of life.

Conclusion: Sufficient long-term results highlight the possiblity of total hip arthroplasty of patients with osteoarthritis even in congenital pubic diastasis. Retroversion of the dysplastic acetabulum, rotation of the posterior part of the pelvis and instability of the pelvic ring in these circumstances might be the most feared aspects in the treatment of these rare cases.

Key words: Congenital pubic diastasis, hip dysplasia, cementless total hip arthroplasty 


\section{Background}

Congenital pubic diastasis in combination with bladder exstrophy is a rather rare malformation. This severe malformation is identified in one out of $30,000-50,000$ live newborns and seen more often in girls. Thereby the genitourinary and musculoskeletal systems will be affected. Numerous osseous morphologic changes are observed in combination with bladder exstrophy. Symphysis diasthasis, rotational anomalies include external rotation of the posterior part of the pelvis and iliac wings and acetabular retroversion are observed in this circumstances (1-3)

In combination with a severe dysplastic hip the treatment of two female patients with hip arthroplasty (THA) in congenital pubic diastasis was so far not described in literature. This prompted us to describe a case, which was treated 9 and 8.5 years ago in our department. Sufficient long-term results highlight the possiblity of total hip arthroplasty in this case. A second case of a dysplastic hip in combination with a symphysis diastasis was treated 6 month ago. Recent examination presented a good clinical success in this special case.

Retroversion of the dysplastic acetabulum, rotation of the posterior part of the pelvis and iliac wings and instability of the pelvic ring in this circumstance might be the most feared aspects in the treatment of THA in these cases $(4,5)$.

\section{Case}

A 35-year female patient 275 pounds of weight with a size of $170 \mathrm{~cm}$ was for the first time presented in our department with severe massive pain in both hips 9 years ago. Mobilization on crutches was just a few steps available. The patient was using a wheelchair even for shorter distances. In prior medical history, a congenital urogenital bladder exstrophy was operated by closed reduction by multiple operations. An external urinary kidney catheter was still in place. Clinical signs show a waddling gait on crutches. The function of both hips in flexion, extension and rotation were massive reduced. X-ray analysis present (Fig.1) a congenital pubic diastasis of $18 \mathrm{~cm}$ with dysplastic hips on both sides. According to the classification of Crowe the disclocation of the femoral head can be measured as grade II (6). A shortening of the anterior ischiopubic segment is obviously. Computed tomography (CT) analysis (Fig.2) present an external rotation of the posterior part of the pelvis and iliac wings combined with 
acetabular retroversion. There was a retroversion of the acetabulum on the right side of $12^{\circ}$, on the left side of $2^{\circ}$. Main problems in these circumstances seems to be the unknown instability concerning the missing anterior ring of the pelvis and the retroverted high-angle, stretched acetabulum on both sides.

Concerning the preoperative planning CT-analysis presented a sufficient depth of the acetabulum to stabilize an acetabular component. By planning the retroverted acetabulum can be compensated by a neutral position of the acetabular cup with elevated liner to avoid femoral head dislocation. Anterior rim reduction helps to avoid impingement problem.

The operation was first admit on the right side in 2011. An osteoconductive cup with high porosity for primary osteointegration (Trabecular Metal ${ }^{\mathrm{TM}}$ modular cup, Zimmer-Biomet $\mathrm{GmbH}$, Winterthur) size $56 \mathrm{~mm}$ was placed with an additional anteverted liner $20^{\circ}$ to maintain stability and avoid dislocation (Fig.3A). Two screws were placed to increase primary stability of the cementless cup in this dysplastic situation. On the femoral side a straight, distally fixed stem Alloclassic-SL (Zimmer-Biomet GmbH, Winterthur) was used to reconstruct anteversion of the proximal femur bone. Operation time was measured with 75 minutes, total blood loss intraoperative was $300 \mathrm{ml}$. A straight lateral approach was used in supine position of the patient.

3 month postoperative the left hip was replaced by a similar procedure. Due to high primary fixation of the cup (TM modular cup) additional screws to enhance fixation were not required (Fig.3b). First follow-up 3 month postoperative presented a pain-free situation, walking on just one stick with a walking distance of more than 100 meter was possible for the affected female patient. There was a definite increase of quality of life and an enhanced walking distance.

With a long-term follow-up of 9 and 8.5 years, the female patient presented a stable situation on the clinical sides. Conventional x-ray control show no signs of loosening of the acetabular or femoral component (Fig.4a, 4b). Heterotopic ossification did not occurred. The Harris Score (7) increased from 14 points to 68 points in a mean postoperative time of 9 years. The patient was pain free by a limited distance walk of 200 meter with one stick on the left side.

In the second case a 52 year old lady had a secondary osteoarthritis affected by severe dysplasia combined with a congenital pubic diastasis $(14.6 \mathrm{~cm})$ (Fig.5 and 6). She had 
multiple prior abdominal operation and an external urinary catheter due to congenital bladder aplasia. By increasing pain on the left hip with decreased walking distance the decision for total hip arthroplasty was been made concerning increasing pain and radiological dysplastic osteoarthritis of the hip. A cementless conventional cup (Allofit, Zimmer-Biomet $\mathrm{GmbH}$, Winterthur) with a metaphyseal fitted stem (Fitmore, Zimmer GmbH, Winterthur) was placed (Fig. 7a,b). 6 months postoperative, the lady patient was free of pain on the affected hip with an increase in quality of life. There was an amelioration of the Harris Hip Score from preoperative 40 points to 80 points.

\section{Discussion}

Congenital pubic diastasis combined with bladder exstrophy is an embryologic malformation resulting in a complex deficit of the anterior midline.

The reason for the development of a bladder exstrophy lies in the incorrect development of the inner abdominal wall, leading to a rupture, so that the urinary bladder is open towards the rear. One can see both ureter openings, from the same urine trickling. This fact results in skeletal and urogenital malformation for the affected patient. In severe cases, the intestinal system is affected with cloacal exstrophy. This clinical picture is a severe congenital malformation identified in one out of 30.000 to 50.000 live newborns and seen more often in girls (1-3).

In some of these cases osseous morphologic changes predominant at the pelvic region can be observed. In regard to the diastasis rotational anomalies include external rotation of the posterior part of the pelvis and iliac wings are presented. All operative procedure affect the problem of pelvic rotation anomalies $(8,9)$.

In literature various procedures to correct the osseous malformation were described so far. Osteotomy types during early childhood have been reported to improve gait and correction of the diastasis (8;9). In adults, a reconstruction of ischio-pubic diastasis is not possible. Due to retroversion of the acetabulum in combination with dysplastic hip malorientation can lead to secondary osteoarthritis of the affected side. 
The procedure of total hip arthroplasty in pubic diastasis is described rarely $(4,5)$. In these case reports the success of the cases were reported by fair manners. The complexity in our described cases are enhanced by the severe dysplasia of the retroverted acetabular grove in both cases. Retroversion of the acetabulum, external rotation of the pelvis and an insufficient acetabular cavity increase the problem of acetabular component fixation. CT can help to analyse the degree of retroversion of the iliac wing, acetabular malorientation and depth of the acetabular cavity. Position of the acetabular component, possible reconstruction of the center of rotation in acetabular grove might be planned with the help of CT-analysis (10)

In the first case we used an osteoconductive trabecular metal cup to enhance stability (11). In the late 90's first biomechanical testing of this material presented a high biomechanical stability to cancellous and cortical bone structure (12). Primary stability and secondary osseointegration will be enhanced by this type of material even in reduced contact to the acetabular bony cavity (13). An anteverted liner can be placed to reduce the dislocation problem. Concerning this circumstance the position of the cup can be placed in a position with a higher inclination angle. On the femoral side a straight rectangle stem was used due to distal fixation philosophy. The antetorsion of the femoral side can be corrected in regard to the position of the stem. The second case had a more normal position of the acetabulum. A conventional cup without screw fixation presented sufficient stability in this case for secondary osseointegration. The key success might be the reconstruction of hip rotation center with the acetabular component even in these cases. In both cases anterior rim trimming was necessary to avoid THA impingement.

\section{Conclusion}

Our experience so far has been rather small in the treatment of these rare cases but the final results in both patients encourage considering total hip arthroplasty. THA is an effective treatment and could improve hip joint function and quality of life in these patients. The midto long-term results are satisfactory. This report needs a multi-center, large sample study to confirm its exact effect in this patient group. 
179 Total hip arthroplasty (THA)

Computed tomography (CT)

Trabecular Metal (TM)

Declarations

Ethics approval and consent for publication Written informed consent was obtained from both patients

Availability of the data and materials Will be confirmed by the authors

Competing interests Both authors declare that they have no competing interests.

\section{Funding Authors None}

\section{$\underline{\text { References }}$}

1. Suson KD, Sponseller PD, Gearhart JP. Bony abnormalities in classic bladder exstrophy: the urologist's perspective. J PediatrUrol. 2013 Apr;9(2):112-22. Epub 2011 Nov 21.

2. Yazici M, Kandemir U, Atilla B, Eryilmaz M.Rotational profile of lower extremities in bladder exstrophy patients with unapproximated pelvis: a clinical and radiologic study in children older than 7 years. J Pediatr Orthop. 1999 Jul-Aug;19(4):531-5.

3. Nordin S, Clementson C, Herrlin K, Haggl und G. Hip configuration and function in bladder exstrophy treated without pelvic osteotomy. J Pediatr Orthop B. 1996 Spring;5(2):119-22. 
4. Drobniewski M, Borowski A, Krasmska M, Sibmski M, Synder M. Hip joint arthroplasty in pubic symphysis separation in the course of congenital bladder exstrophy - study of two cases. Ortop Traumatol Rehabil. 2016 Aug 30;18(4):367-373

5. Camera A, Grappiolo G, Santoro G. Hip arthroplasty in a patient with congenital pubic diastasis and bladder exstrophy. Hip Int. 2009 Apr-Jun;19(2):155-6

6. Crowe JF, Mani VJ, Ranawat CS. Total hip replacement in congenital dislocation and dysplasia of the hip. J Bone Joint Surg Am. 1979; 61:15-23

7. Harris WH. Traumatic arthritis of the hip after dislocation and acetabular fracture: treatment of mold arthroplasty. An end result study using a new method of end result evaluation. J Bone Joint Surg (Am) 1969:737-755

8. Sponseller PD, Jani MM, Jeffs RD, Gearhart JP. Anterior innominate osteotomy in repair of bladder exstrophy. J Bone Joint Surg Am. 2001Feb;83(2):184-93.

9. Nhan DT, Sponseller PD. Bilateral anterior innominate osteotomy for bladder Exstrophy. JBJS Essent Surg Tech, 2019, 9

10. Imai H, Miyawaki J, Kamada T, Tabeka J, Mashima N, Miura H. Preoperative planning and postoperative evaluation of total hip arthroplasty that takes combined anteversion. Eur J Orthop Surg Traumatol 2016; 26:493-500

11. Laaksonen I, Lorimer M, Gromov K, Eskelinen A, Rolfson O, Graves SE, Malchau H, Mohaddes M. Trabecular metal acetabular components in primary total hip arthroplasty. Acta Orthop. 2018 Jun;89(3):259-264.

12. Zhang $Y$, Ahn PB, Fitzpetrick DC, Heiner AD, Poggie R, Brown TD. Interfacial friction behavior: Cancellous bone, cortical bone, and a novel porous tanatlum biomaterial. Journal of musculoskeletal research 1999: 245-251 
234 13. Perticarini L, Zanon G, Rossi S., Benazzo F. Outcomes of a Trabecular Titanium ${ }^{\mathrm{TM}}$

235 Acetabular Component in Hip Arthroplasty: Results at Minimum 5 Years Follow-Up. BMC

236 Musculoskelet Disord 2015,16:375

237

238

239 


\section{Figures}

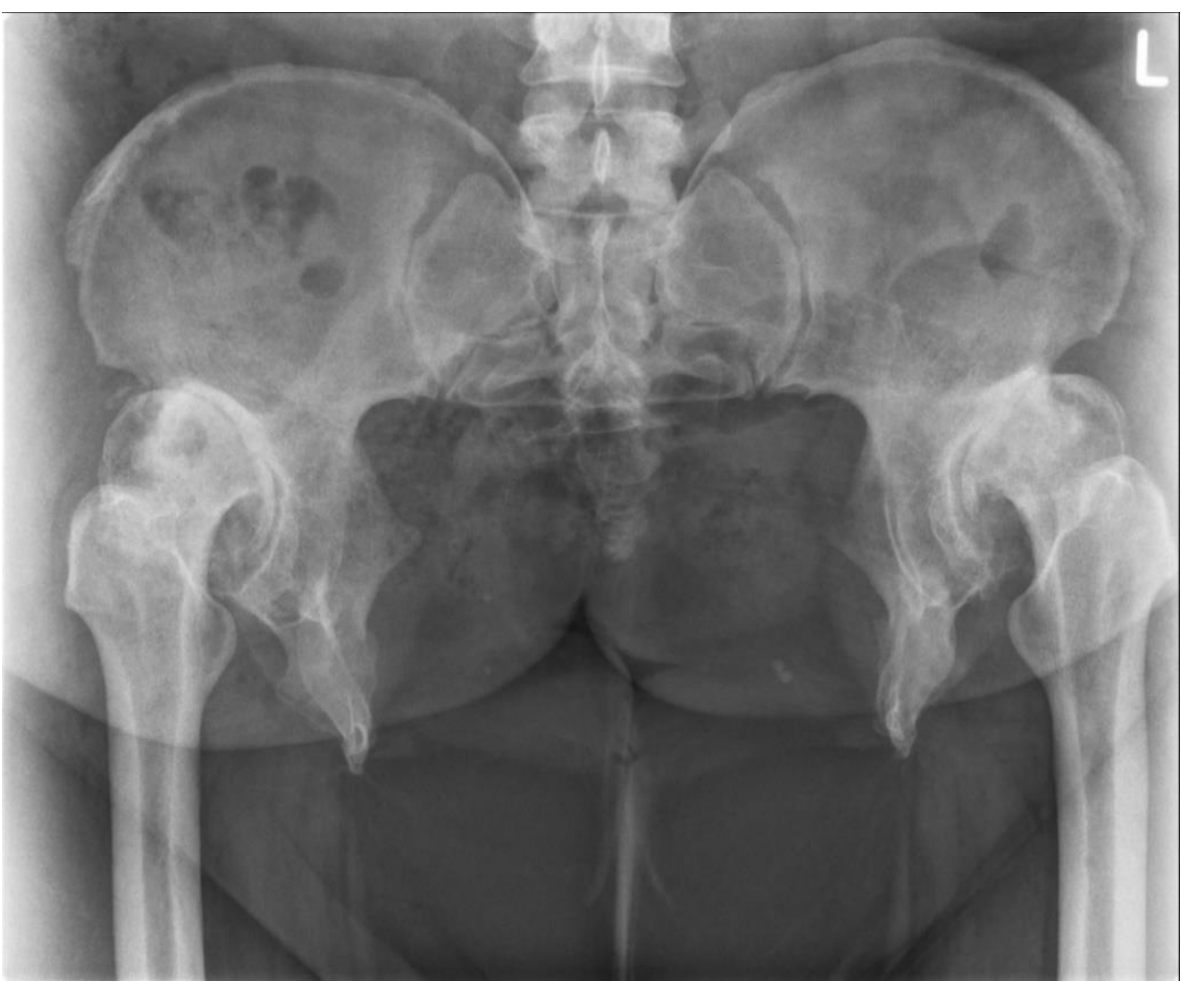

Fig.1 X-ray analysis present a congenital pubic diastasis of $18 \mathrm{~cm}$ with severe dysplastic hips on both sides in a female patient age 35 years.

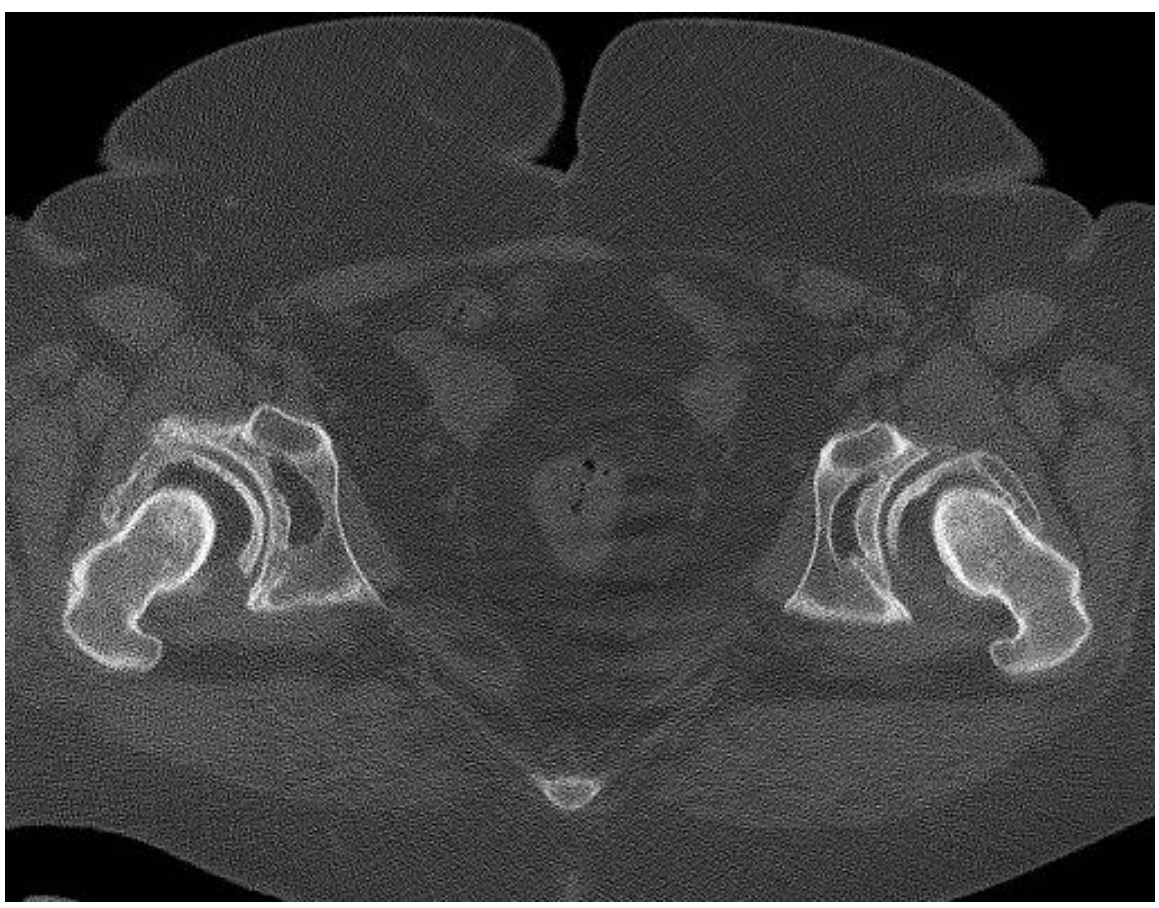

Fig.2 Computed tomography (CT) analysis present an acetabular retroversion combined with external rotation of the posterior part of the pelvis and iliac wings 


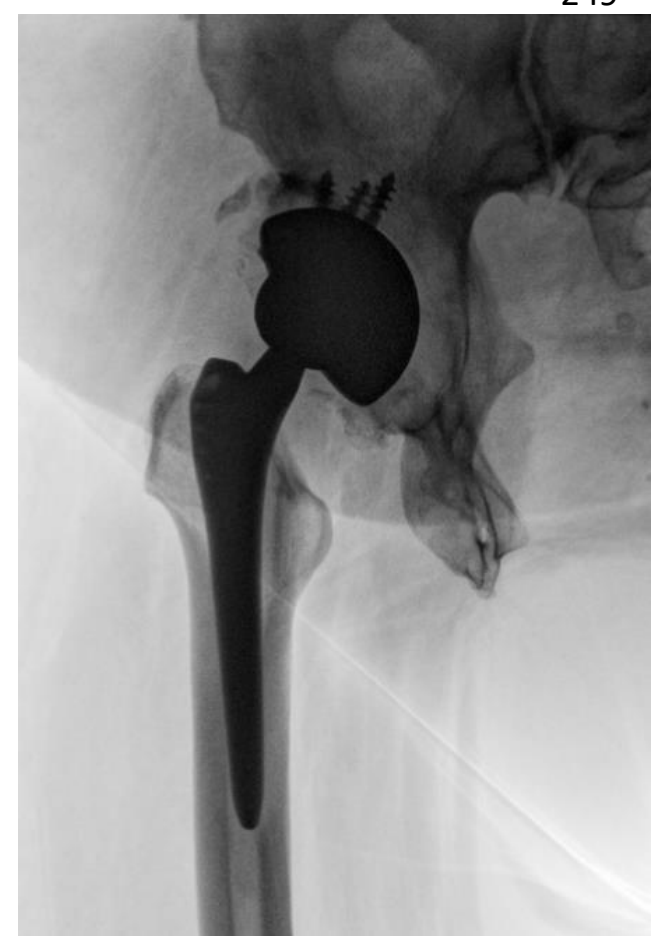

261 Fig.3a Postoperative $x$-ray on right hip with an osteoconductive cup (TM modular cup) size $26256 \mathrm{~mm}$ with an additional anteverted liner $20^{\circ}$ combined with distally fixed stem (Alloclassic$263 \mathrm{SL})$

264

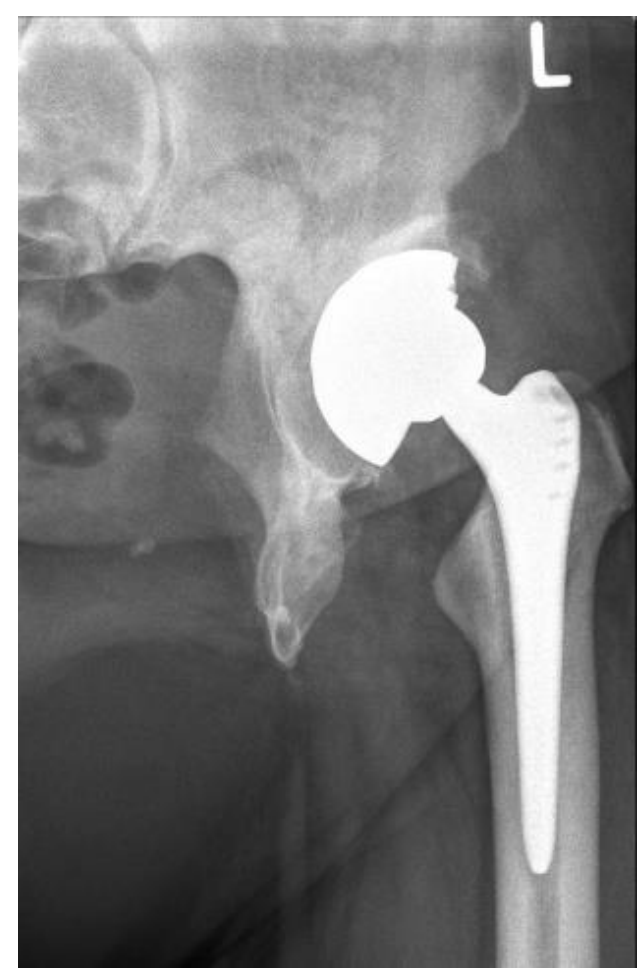

Fig.3b Postoperative $x$-ray on the left hip 3 month 


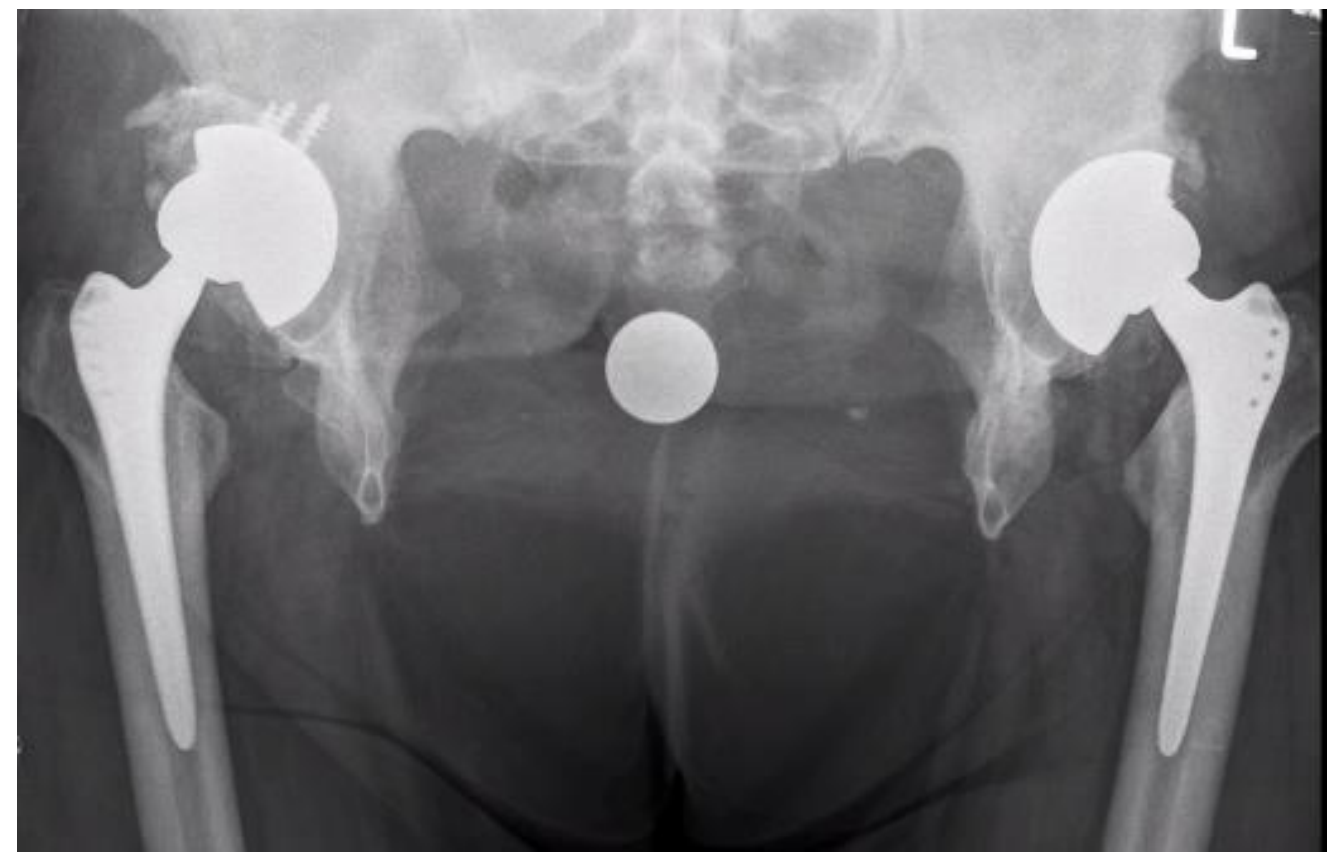

Fig. 4a 9 years postoperative x-ray control show no signs of loosening on the acetabular or femoral component

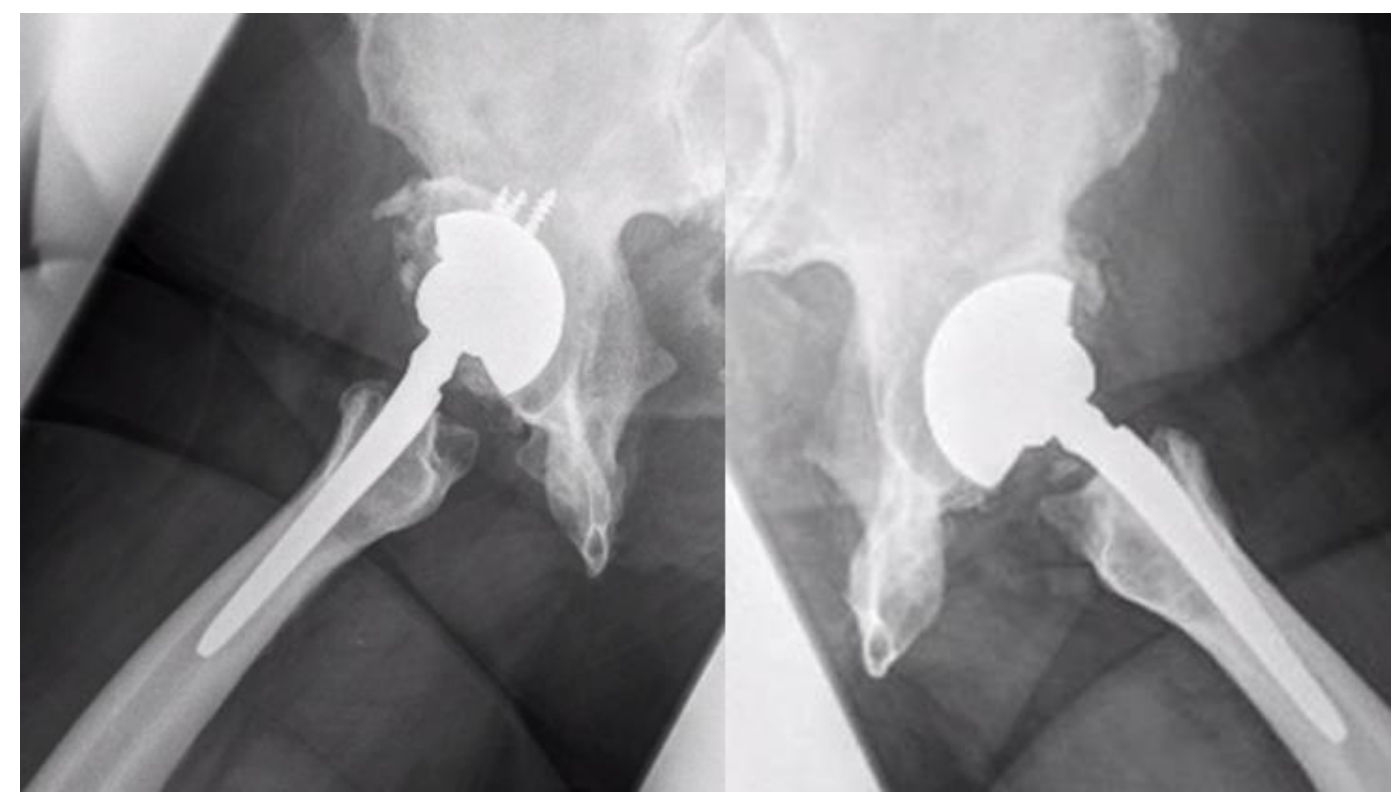

Fig.4b 9 years postoperative $x$-ray control in lateral approach present no signs of loosening of the component 


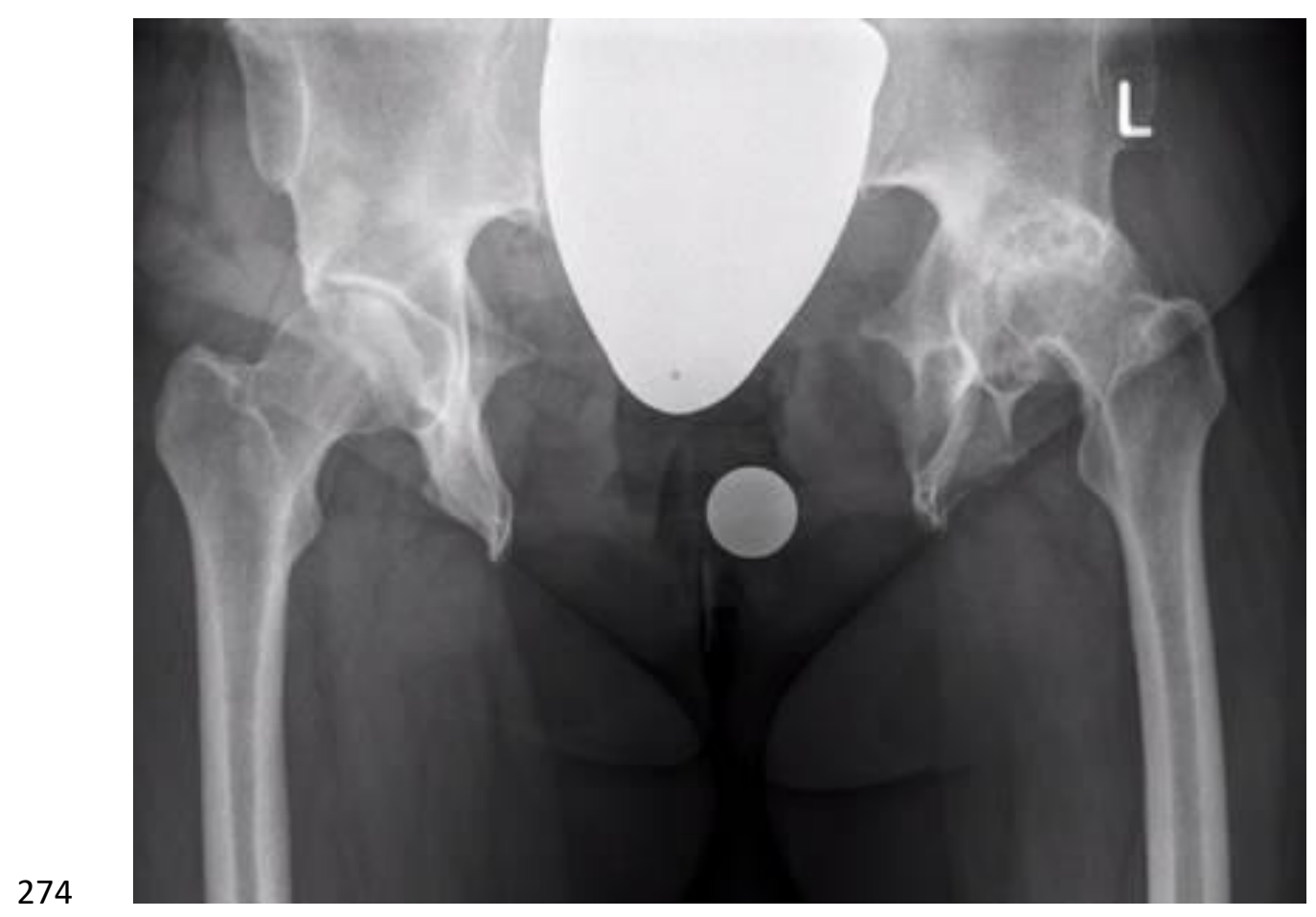

275 Fig.5 X-ray in a patient age 52 with left osteoarthritis by severe dysplasia combined with a congenital pubic diastasis $(14.6 \mathrm{~cm})$

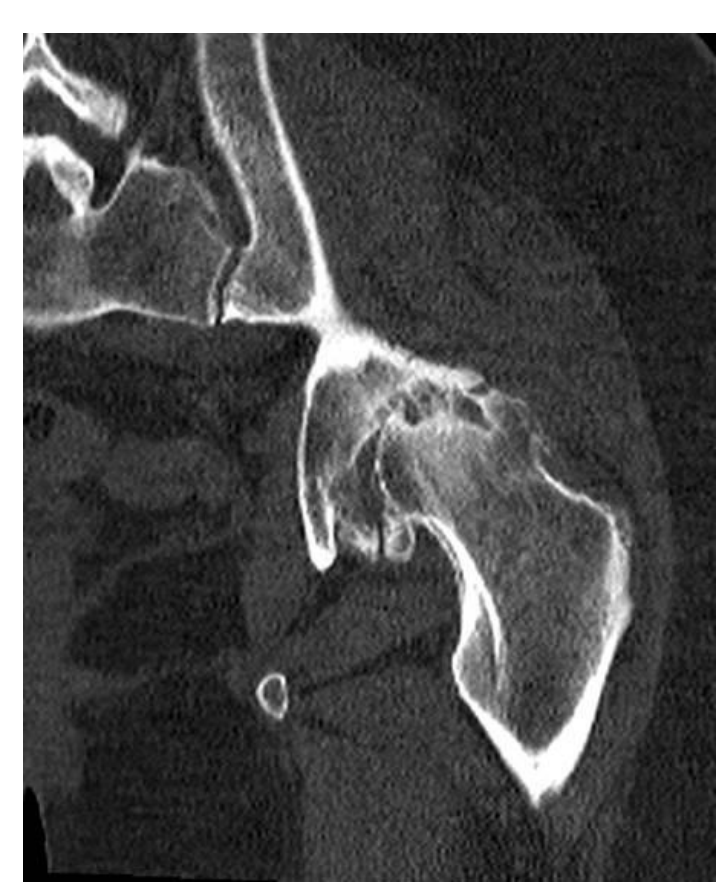

Fig.6 Computed tomography with osteoarthritis in regard to dysplasia 

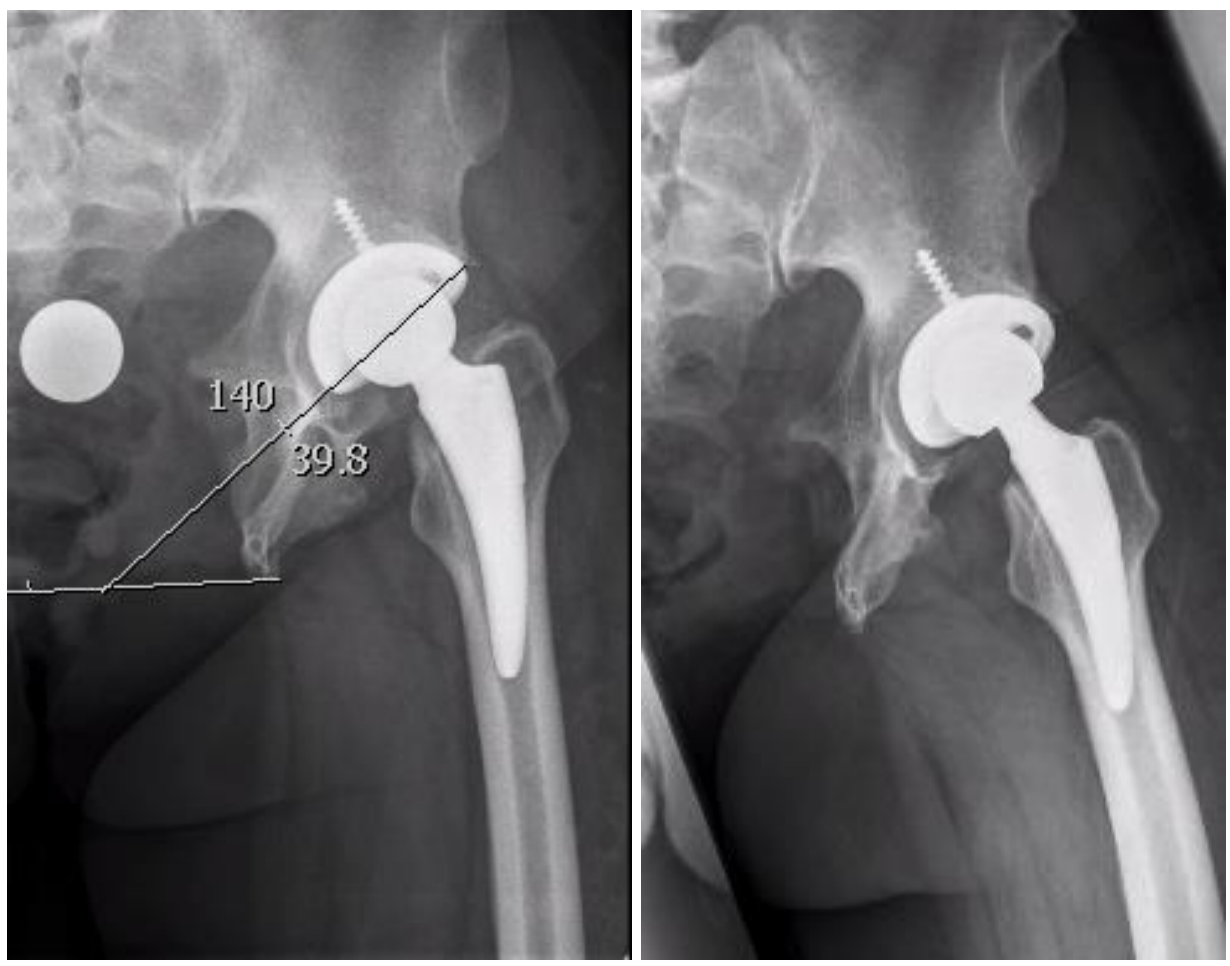

Fig.7a,b Postoperative x ray; conventional cup (Allofit) with a metaphyseal fitted stem

(Fitmore) was placed in this case 
Figures

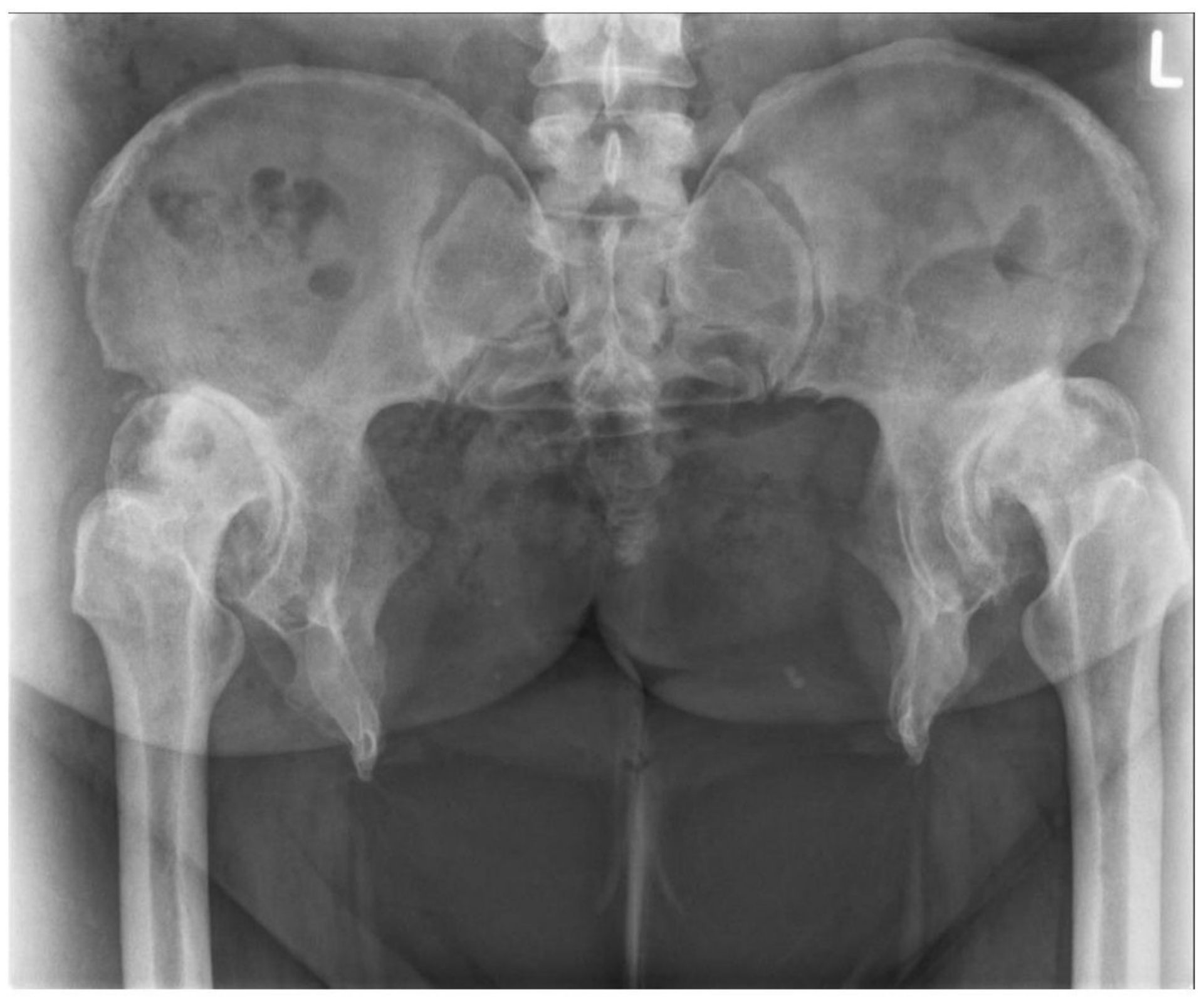

\section{Figure 1}

X-ray analysis present a congenital pubic diastasis of $18 \mathrm{~cm}$ with severe dysplastic hips on both sides in a female patient age 35 years. 


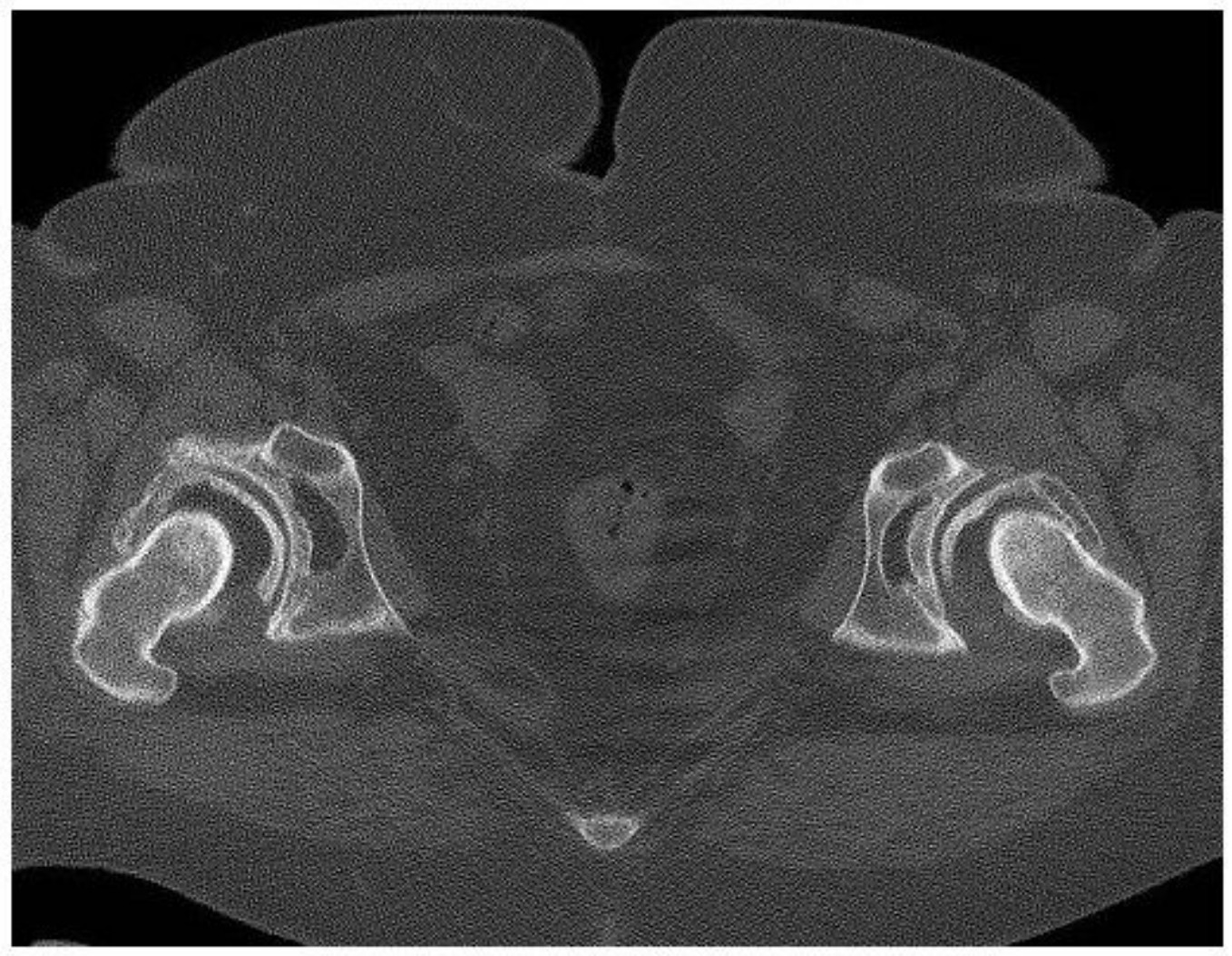

Figure 2

Computed tomography (CT) analysis present an acetabular retroversion combined with external rotation of the posterior part of the pelvis and iliac wings 

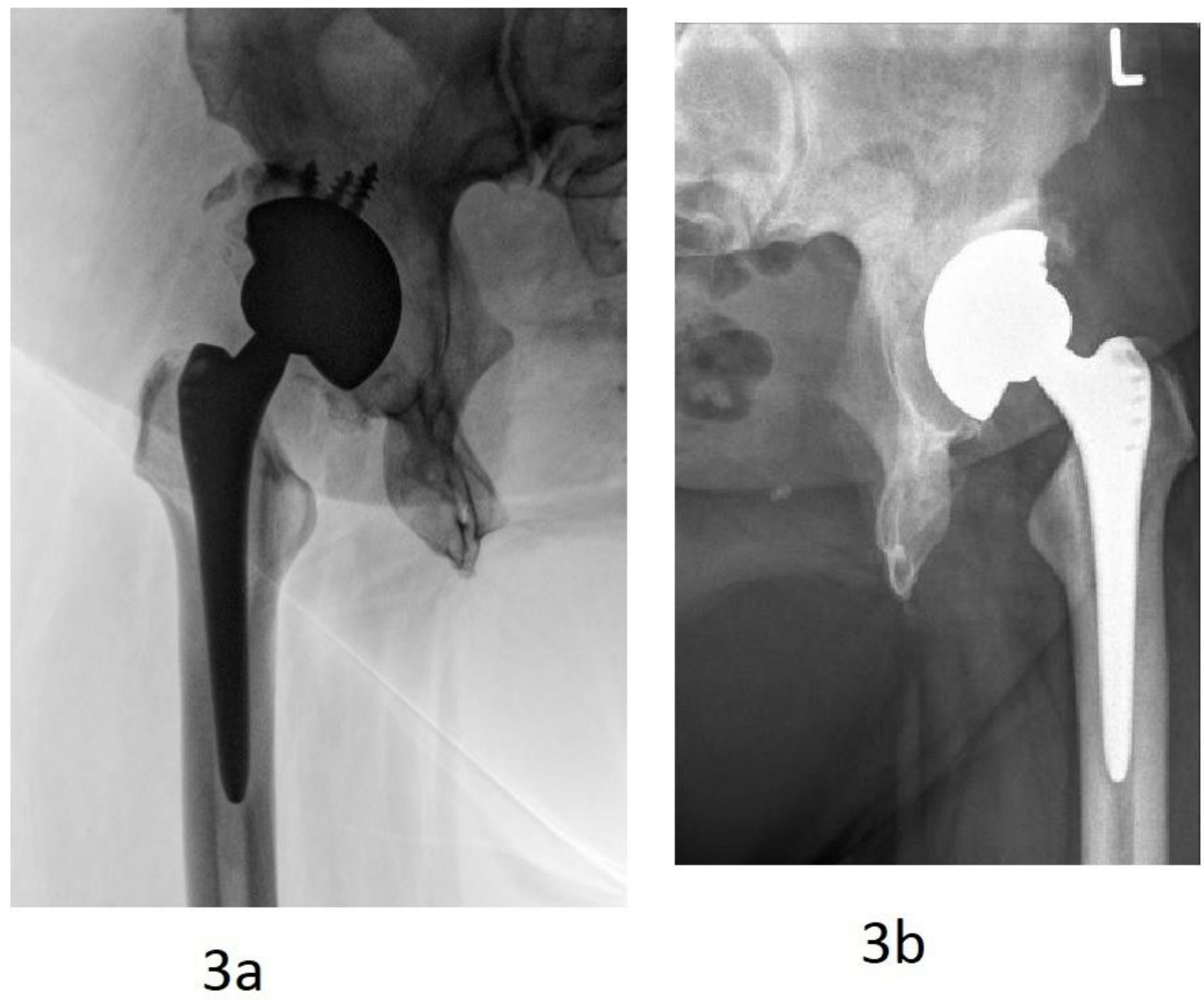

$3 b$

\section{Figure 3}

a. Postoperative x-ray on right hip with an osteoconductive cup (TM modular cup) size $56 \mathrm{~mm}$ with an additional anteverted liner $20^{\circ}$ combined with distally fixed stem (Alloclassic-SL) b. Postoperative x-ray on the left hip 3 month 


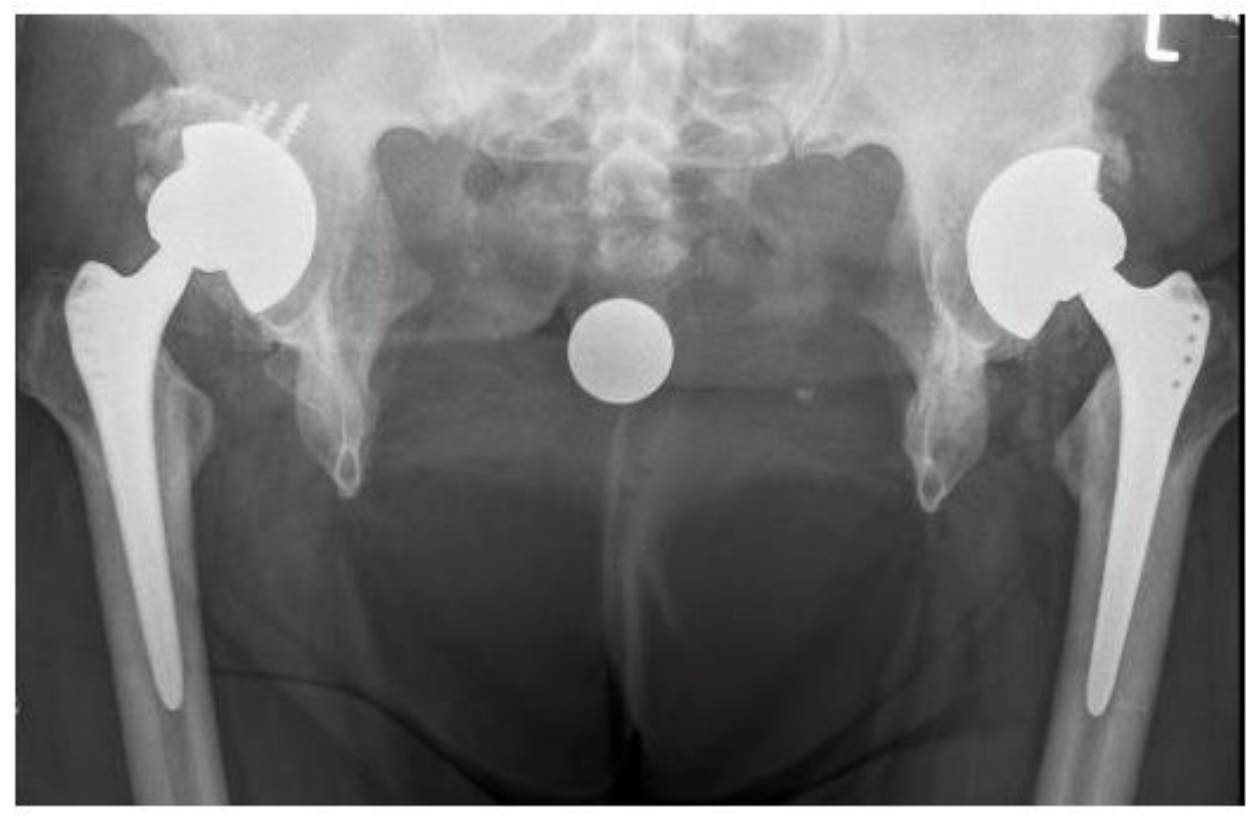

\section{$4 a$}

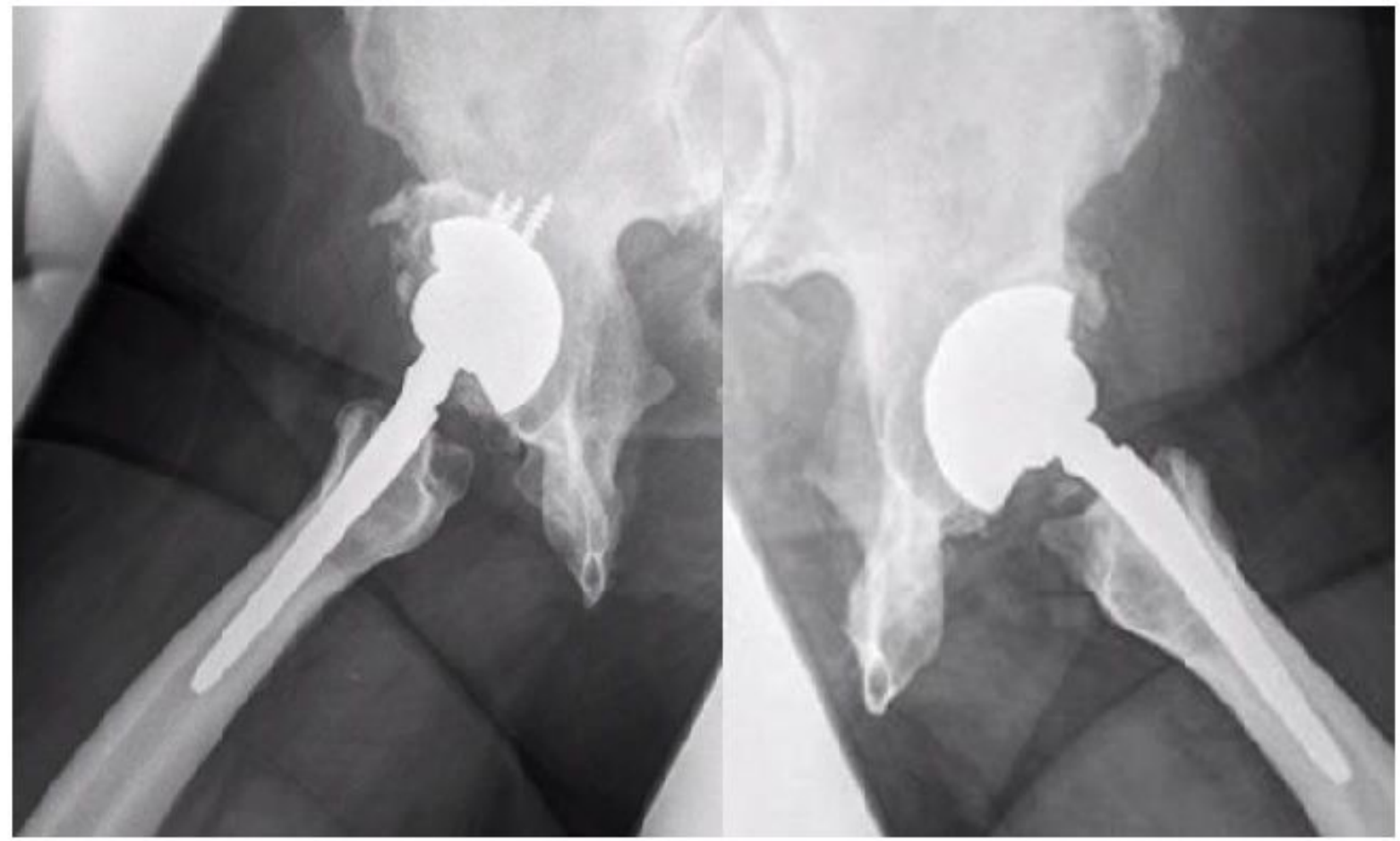

\section{$4 b$}

Figure 4

a. 9 years postoperative $x$-ray control show no signs of loosening on the acetabular or femoral component b. 9 years postoperative $x$-ray control in lateral approach present no signs of loosening of the component 


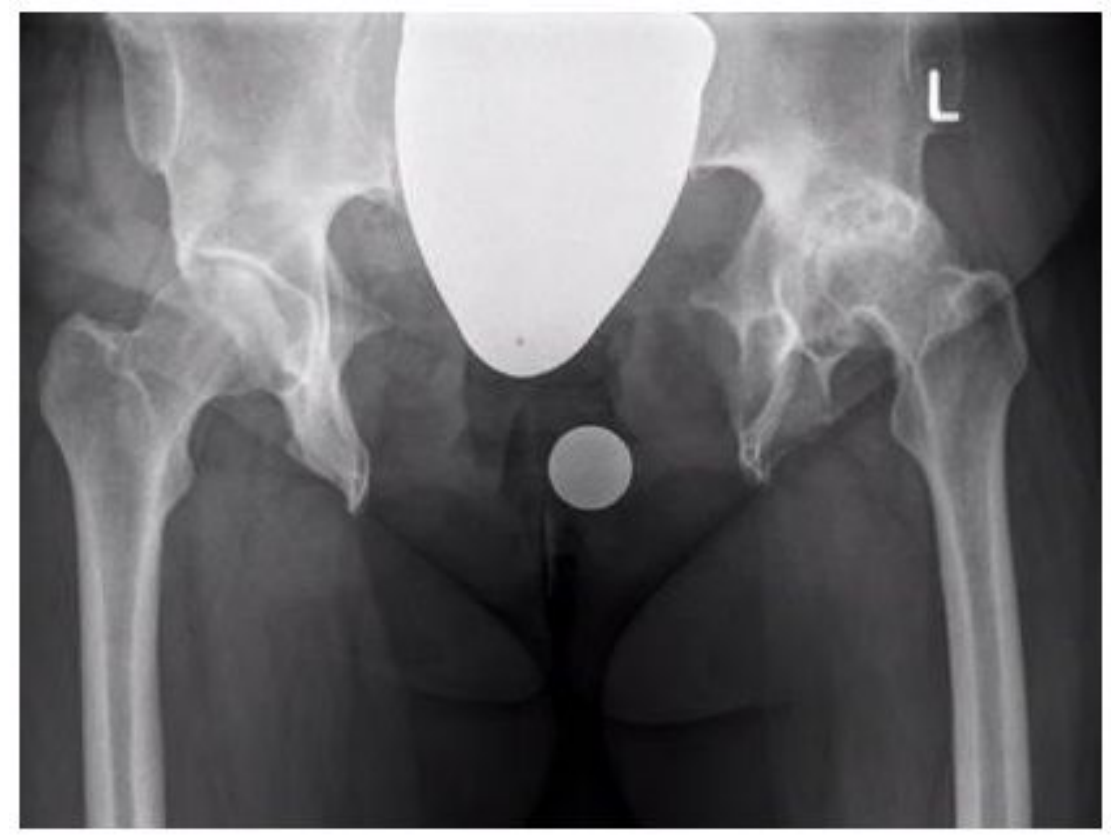

Figure 5

X-ray in a patient age 52 with left osteoarthritis by severe dysplasia combined with a congenital pubic diastasis $(14.6 \mathrm{~cm})$ 


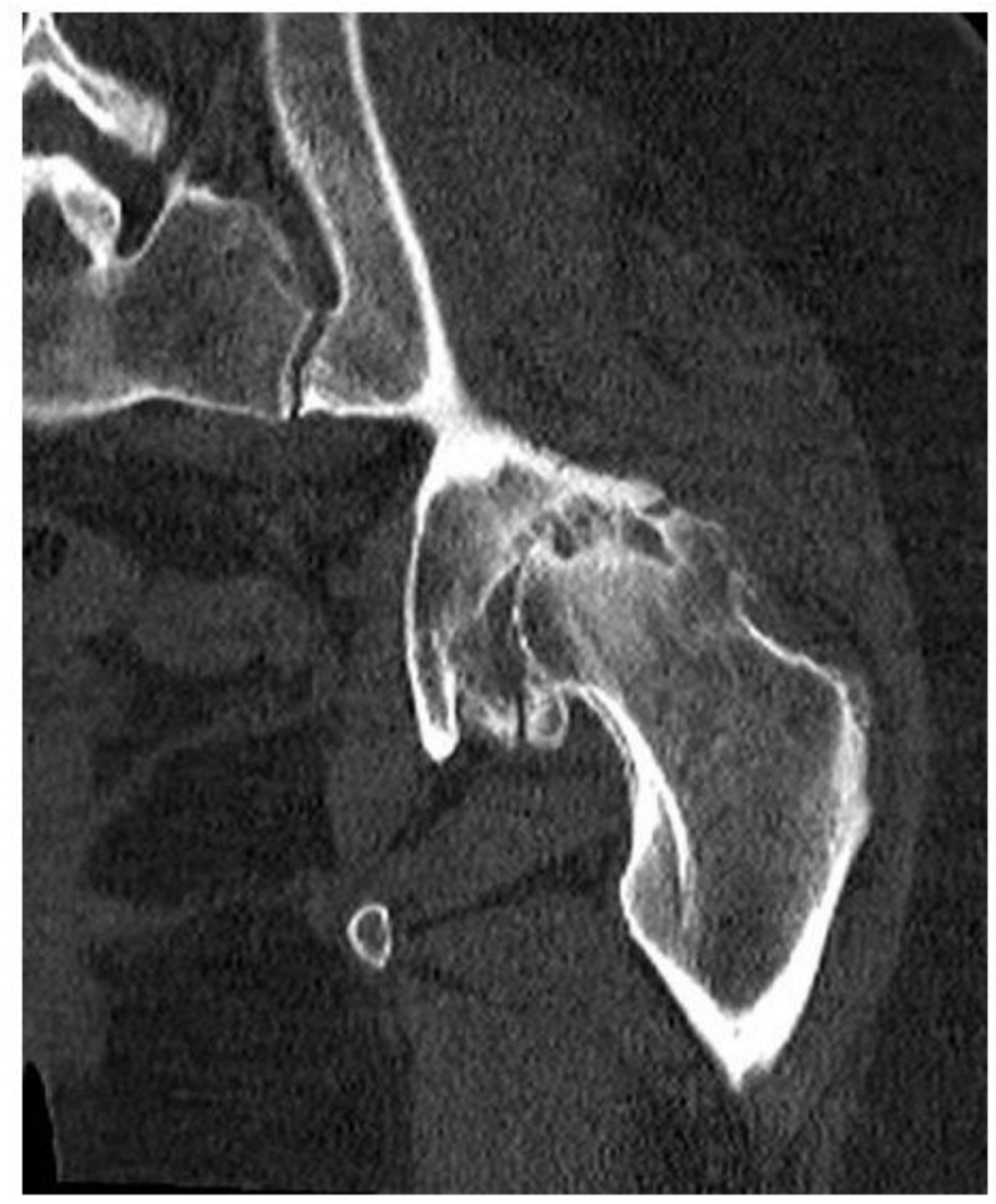

Figure 6

Computed tomography with osteoarthritis in regard to dysplasia 


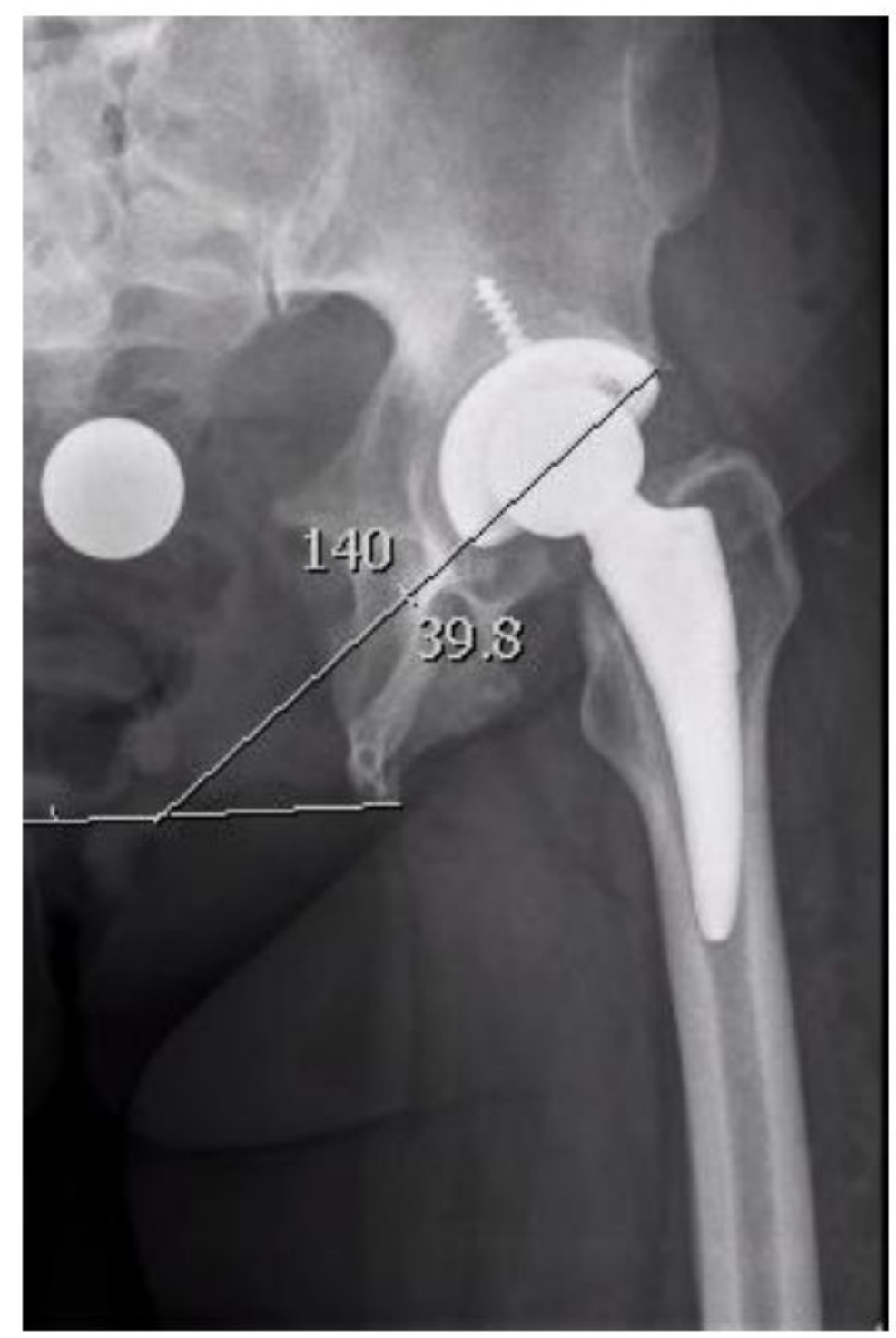

$7 a$

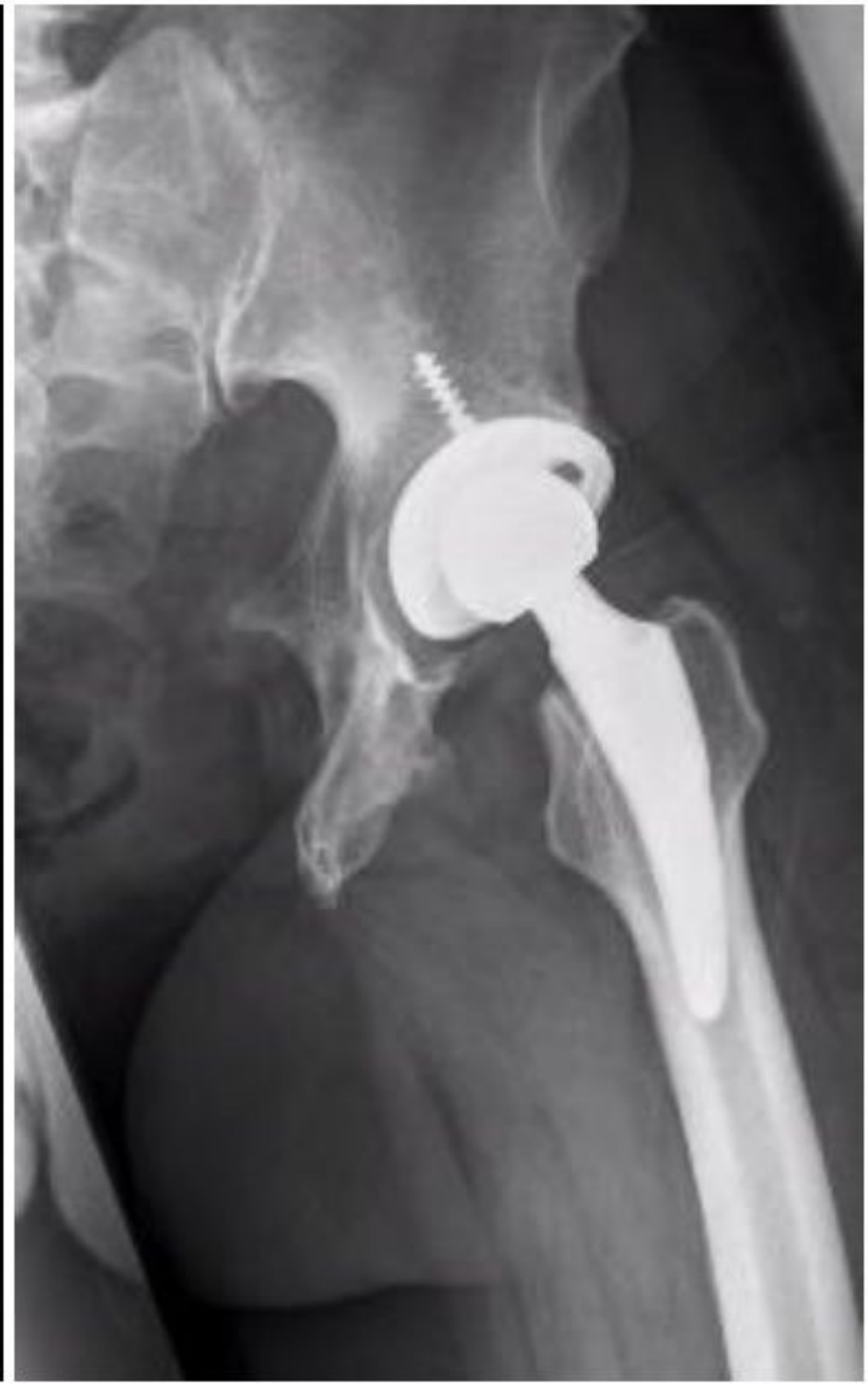

$7 \mathrm{~b}$

\section{Figure 7}

a,b Postoperative $x$ ray; conventional cup (Allofit) with a metaphyseal fitted stem (Fitmore) was placed in this case 